\title{
The potential role of epigenetic modulations in BPPV maneuver exercises
}

\author{
Kun-Ling Tsai ${ }^{1}$, Chia-To Wang ${ }^{2,3}$, Chia-Hua Kuo ${ }^{4}$, Yuan-Yang Cheng ${ }^{3,5,7}$, Hsin-I Ma ${ }^{6}$, \\ Ching-Hsia Hung ${ }^{1}$, Yi-Ju Tsai ${ }^{1}$ and Chung-Lan Kao ${ }^{2,7}$ \\ ${ }^{1}$ Department of Physical Therapy, College of Medicine, National Cheng Kung University, Tainan, Taiwan \\ ${ }^{2}$ Department of Physical Medicine and Rehabilitation, Taipei Veterans General Hospital, Taipei, Taiwan \\ ${ }^{3}$ Institute of Clinical Medicine, School of Medicine, National Yang-Ming University, Taipei, Taiwan \\ ${ }^{4}$ Department of Sports Sciences, University of Taipei, Taipei, Taiwan \\ ${ }^{5}$ Department of Physical Medicine and Rehabilitation, Taichung Veterans General Hospital, Taichung, Taiwan \\ ${ }^{6}$ Department of Neurological Surgery, Tri-Service General Hospital, National Defense Medical Center, Taipei, Taiwan \\ 7 Department of Physical Medicine and Rehabilitation, School of Medicine National Yang-Ming University, Taipei, Taiwan \\ Correspondence to: Chung-Lan Kao, email: clkao@vghtpe.gov.tw \\ Keywords: benign paroxysmal positional vertigo, microRNA, Sirtuin 1, maneuver exercises, Gerotarget \\ Received: March 04, $2016 \quad$ Accepted: May 10, $2016 \quad$ Published: May 18, 2016
}

\section{ABSTRACT}

Benign paroxysmal positional vertigo (BPPV) is one of the most common complaints encountered in clinics and is strongly correlated with advanced age or, possibly, degeneration. Redistribution exercises are the most effective approaches to treat BPPV, and canalith repositioning procedure (CRP) cure most BPPV cases. However, the mechanisms through which the treatment modulates systemic molecules in BPPV patients remain largely unknown. In this study, we report that the miR-34a and Sirtuin 1 (SIRT1) genes correlated with the treatment effects of CRP in BPPV subjects. We found that miR-34a expression was largely inhibited and SIRT1 expression was significantly reversed after BPPV maneuver treatment. We also confirmed that the PPAR-y, PGC-1 and FoxO gene expressions were decreased immediately after canalith repositioning procedure (CRP) for BPPV, and were largely increased after a complete cure of BPPV. Moreover, we observed that after a complete recovery of $B P P V$, the ROS concentrations, pro-inflammatory cytokine concentrations and p53 expression levels were attenuated. We conclude that BPPV treatment might involve some epigenetic regulations through the mediation of miR-34a, SIRT1 functions and repression of redox status.

\section{INTRODUCTION}

Benign paroxysmal positional vertigo (BPPV) is one of the most common complaints encountered in otology, neurology and rehabilitation clinics. The typical presentation of BPPV is intense, brief vertigo spells that are provoked by a change in head position [1]. Benign paroxysmal positional vertigo is believed to be the consequence of displaced otoconia within the semicircular canals located in the inner ear [2]. Individuals with a history of head trauma or ear infections are prone to developing BPPV [3]. Moreover, BPPV occurrence is highly related to advanced age or, possibly, degeneration. One previous study suggested that age, migraines, hypertension, hyperlipidemia and stroke are independently associated with BPPV [4]. The occurrence and recurrence rates are increased among the senior population [5].

Repositioning maneuvers are effective interventions for BPPV. Brandt and Daroff designed the first operative physical therapy of redistribution exercise [6]. The development of redistribution exercise was based on the theory of cupulolithiasis, which explains the mechanism of BPPV as calcium deposits adhering to cupula. The Brand-Daroff redistribution maneuver exercise 
cured approximately $92.68 \%$ of BPPV patients, with a recurrence rate of approximately 4.22\% [7]. Another maneuver exercise, modified by Dr. Epley et al., the socalled canalith repositioning procedure (CRP), has been extensively used for the treatment of posterior canal BPPV [8]. The canalith repositioning procedure is based on the canalolithiasis theory, which indicates that BPPV is caused by free-floating debris in the ampullofugal branches of semicircular canals. The free-floating debris moves to the most dependent part of the semicircular canals relative to gravity during head movements, causing transient spells of vertiginous episodes [1].

With the advancement of biomolecular medicine, disease etiologies can be investigated more thoroughly by exploring the underlying cellular pathways. microRNAs (miRNAs) have been found circulating in human blood, particularly in cell-free plasma. miRNAs play vital roles in the regulation of pathological communications. Importantly, in human blood, circulating plasma miRNAs appear to be secreted from the cellular compartment [9]. miR-34a, one well-known miRNA involved in cell cycle regulation and p53-related apoptosis signaling transduction, was first revealed as a posttranscriptional regulator of SIRT1 [10]. Silencing of miR-34a function by antisense oligonucleotides attenuates p53 acetylation and PUMA expression at the protein level [11]. miR34a mediates human endothelial cell pro-senescence responses, increases the fraction of cells in G1 phase of the cell cycle and impedes angiogenesis by modulating SIRT1 and forkhead box O transcription factor 1 (FoxO1) [12]. Shephali et al. revealed that miR-34a expression is enriched in circulating blood plasma in Alzheimer's disease [13]. Julien et al. also demonstrated that SIRT1 reduction parallels the accumulation of tau in Alzheimer's disease, suggesting that miR-34a and SIRT1 play important roles in the progression of neurodegenerative diseases.

Vast amounts of literature have indicated that BPPV increases with advanced age . Recent knowledge implied BPPV might be a results of otconia degeneration [14]. However, the mechanisms behind this degeneration is still unclear. Pro-inflammatory regulators are activated during the aging process. NF- $\kappa \mathrm{B}$ is one of the most widely known mediators controlling pro-inflammatory signaling [15]. Chronic inflammation occurs in the brain of the senescence-accelerated (SAMP8) mouse, and it constitutively activates pro-inflammatory genes through NF- $\kappa \mathrm{B}$ up-regulation [16]. Cyclooxygenase-2 (COX-2) is an important enzyme in the prostaglandin (PG) synthetic cascade during the aging process [17]. Prostaglandin E2 (PGE2) secretion was found to be up-regulated in aged mice (23 months old) only, which provides some knowledge regarding the senescence-related proinflammatory process [18]. It has also been shown that signaling transduction via the FoxO longevity factors and SIRT1 can functionally mitigate the NF- $\kappa$ B pathway and simultaneously repress the inflammation-aging process [19]. Currently, repositioning maneuver exercises are believed to be the only effective non-pharmacological interventions that can cure BPPV through mechanical ways [20]. The ultimate goal of these maneuvers is to reposition the otoconia into the cupula. Whether these maneuvers involve biomolecular mechanisms, such as inflammation and senescence, has not yet been reported.

The primary aim of this study was to investigate the epigenetic regulation of miR-34a expression in circulating blood samples after CRP in patients with the canalolithiasis type of posterior canal BPPV. Because the SIRT1 gene is highly related to aging and neurodegenerative diseases [21], we also hypothesize that the expression of the SIRT1 gene, which is directly regulated by miR-34a, along with the SIRT1 downstream genes would be positively activated after complete treatment for BPPV.

\section{RESULTS}

\section{Subject characteristics}

A total of $20 \mathrm{BPPV}$ patients (mean age $=62.8 \pm$ 14.4; $\mathrm{M}: \mathrm{F}=3: 17$; left posterior canal $\mathrm{BPPV}=5$, right posterior canal BPPV $=15$ ) completed treatment and blood sampling for this study. The duration for complete resolution of BPPV was $8.53 \pm 5.27$ days. During the follow up visits, all patients had symptomatic relief and were free from positional vertigos. Using infra-red video goggles, no nystagmus was observed in Dix-Hallpike positions.

\section{Patient miR-34a and SIRT1 expression levels were altered upon completion of BPPV treatment}

miR-34a plays an important role in the regulation of neurodegenerative diseases [13]. Moreover, Li's work confirmed concordant increases in miR-34a expression in the brain and plasma. Li et al. concluded that circulating miR34a was an RNA based, noninvasive biomarker for brain aging [10]. Our previous publication reported that BPPV was positively related to aging [5]. Therefore, we investigated miR-34a levels in plasma samples using quantitative reverse transcription PCR (qPCR) analyses. As shown in Figure 1A, miR-34a expression was relatively higher in the blood samples of the BPPV attacked patients. However, miR-34a expression was significantly reduced after CRP treatment. SIRT1 is an important protective factor against neurodegenerative diseases in humans. A decrease in SIRT1 function has been reported to occur with increasing age [22]. Figure 1B shows that blood SIRT1 expression levels were relatively repressed in BPPV samples; however, the expression pattern was reversed following a BPPV cure $(P<0.05)$. 


\section{DBC1 expression level was affected in clinical treatment}

Deleted in breast cancer-1 (DBC1) was firstly described from the observation that $\mathrm{DBC} 1$ is deleted in some human breast cancers [23]. DBC1 directly interacted with the catalytic domain of SIRT1, thereby inhibiting
SIRT1 activities [24]. Moreover, DBC1 is thought to be one key co-factor for the mediation of the IKK- $\beta$-NF- $\mathrm{KB}$ signaling pathway [25]. DBC1/SIRT1 complex may act an important role in regulation of aging and inflammatory responses. Figure 2A and 2B shows DBC1 mRNA and protein expression levels were attenuated following a BPPV cure $(P<0.05)$.
(A)

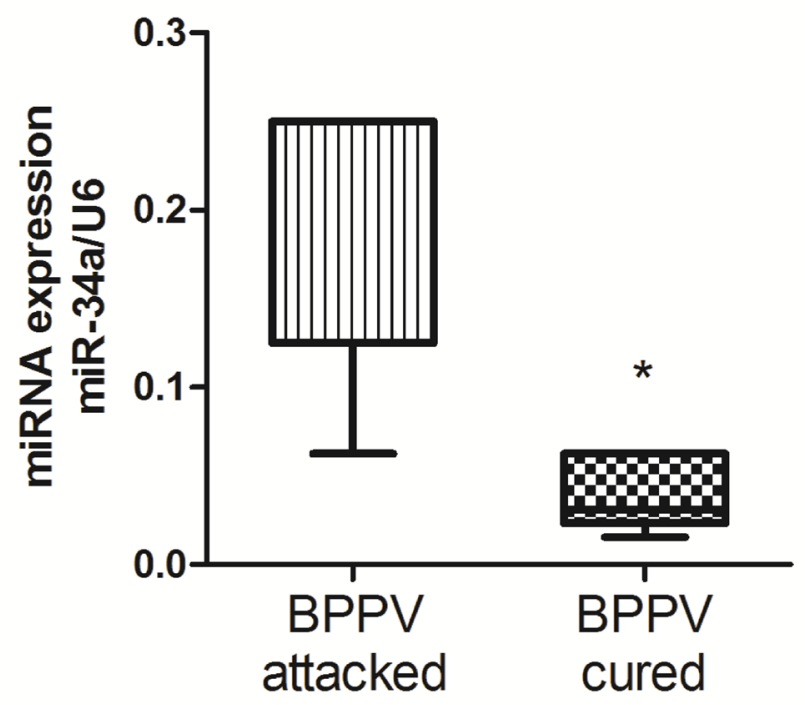

(B)

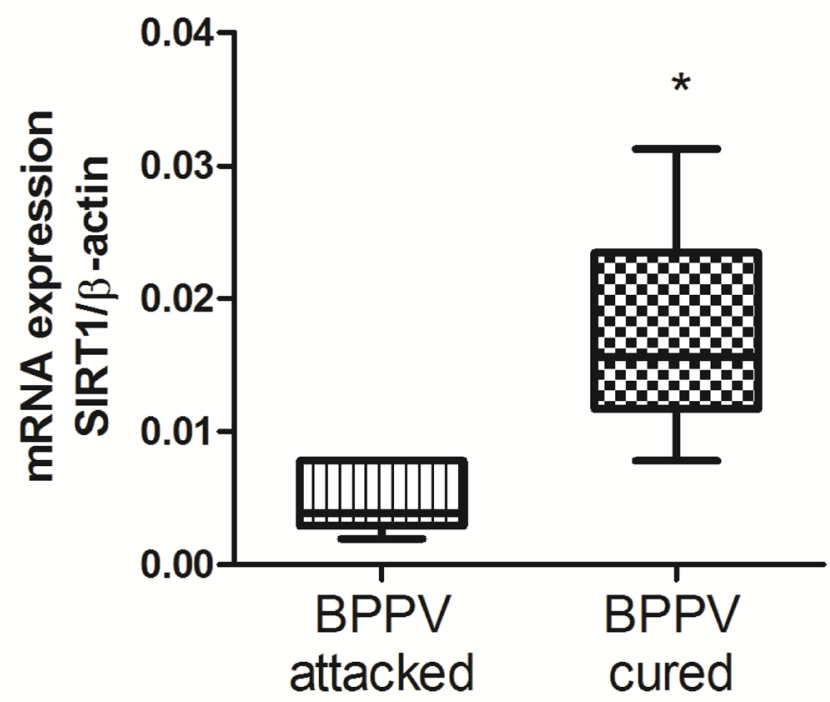

Figure 1: Expression levels of A. miR-34a and B. SIRT1 in BPPV-attacked and BPPV-cured patients after treatment of maneuver exercise. Total blood mRNAs were isolated from patients, the miR-34a and SIRT1 expression were investigated after maneuver exercise intervention using real-time PCR.

(A)

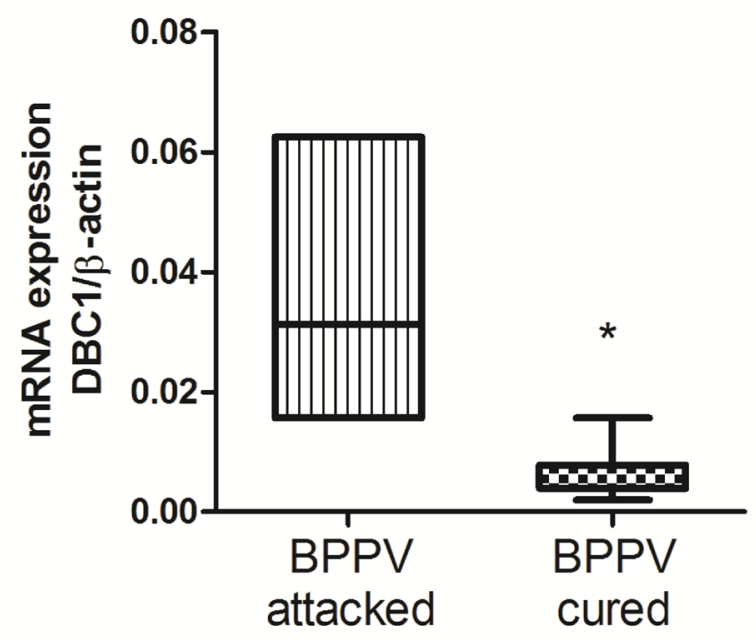

(B)

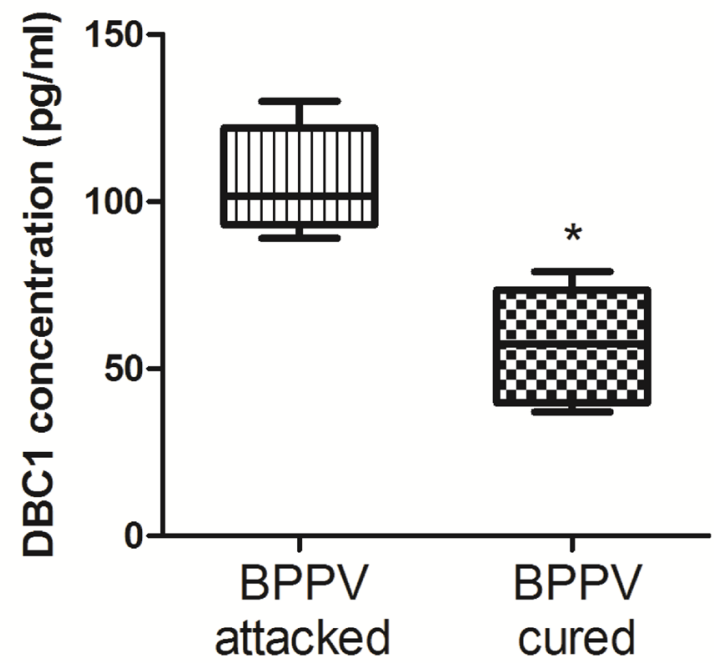

Figure 2: Expression levels of DBC1 in BPPV-attacked and BPPV-cured patients after treatment of maneuver exercise. A. Total blood mRNAs from BPPV-attacked and BPPV-cured patients were isolated from patients, and the DBC1expression was tested after maneuver exercise intervention using real-time PCR. B. Blood plasma was isolated from patients. DBC1 concentration was investigated using ELISA kits. 
Following the canalith repositioning procedure, p53, PGC-1 and PPAR- $\gamma$ expression levels were changed

miR-34a functions in the SIRT1-mediated deacetylation of p53 and in the neuronal-protective signaling pathway [26]. Moreover, SIRT1/p53/miR-34a is involved in a positive feedback loop involved in the regulation of miR-34a expression. Indeed, up-regulated miR-34a mitigates SIRT1 function, and attenuates p53 deacetylation, leading to an increase in acetylated p53 transcriptional activity [11]. We previously suggested that miR-34a expression were reduced and the SIRT1 expression level were up-regulated after clinical BPPV rehabilitation. We turned our attention on investigating the p53 level in blood samples of BPPV patients immediately after (BPPV attacked) and 1-2 weeks after

(A)

(C)
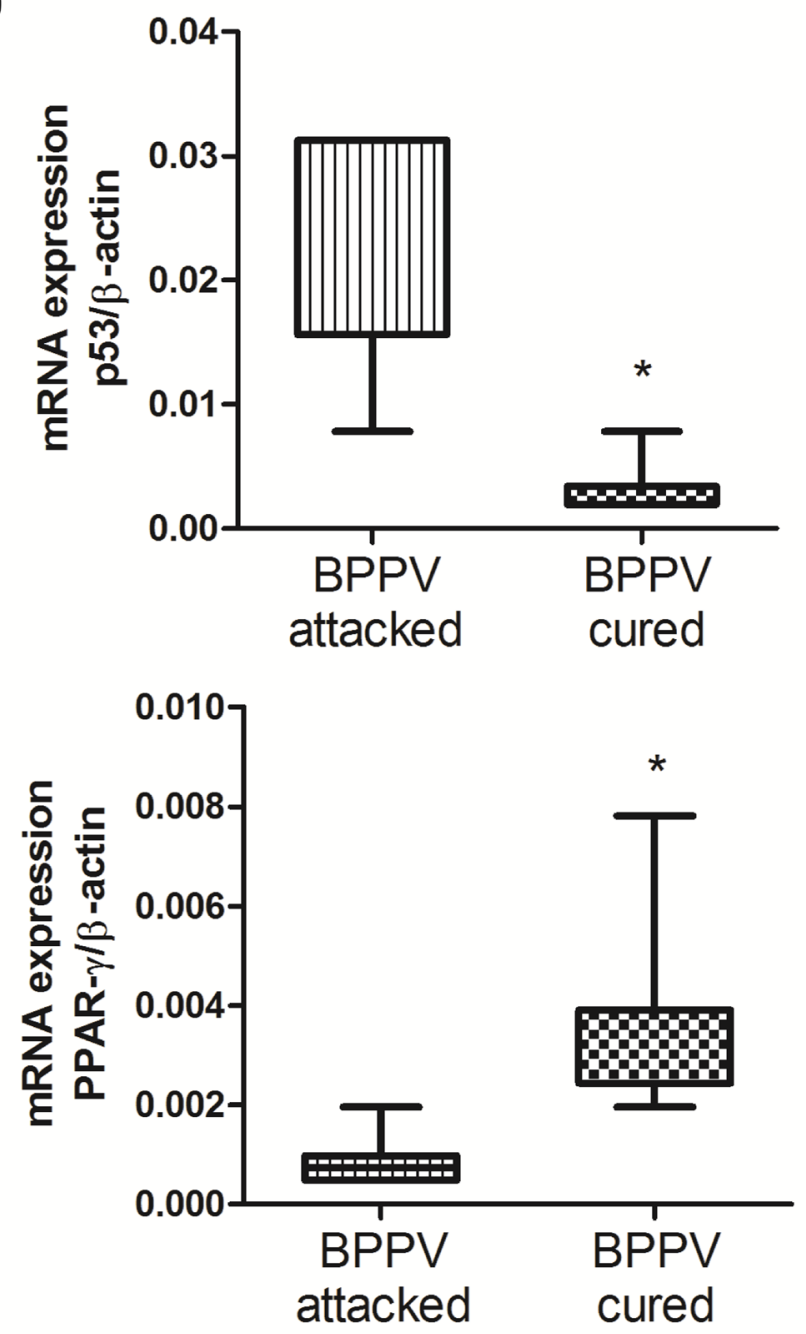

(BPPV cured) CRP treatment. As shown in Figure 3A, using real-time PCR, we confirmed that p53 mRNA levels were significantly higher in the BPPV samples compared with the BPPV-cured samples $(P<0.05)$. PPAR- $\gamma$ coactivator- $1 \alpha$ (PGC-1 $\alpha)$ and peroxisome proliferatoractivated receptor-gamma (PPAR- $\gamma$ ) are important SIRT1 downstream target genes [27]. We used real-time PCR to further confirm whether the gene expression of PGC-1 $\alpha$ and PPAR- $\gamma$ was involved in BPPV episodes. The data shown in Figure 3B and 3C suggested that the blood expression levels of PPAR- $\gamma$ and PGC- 1 were significantly lower in BPPV attacked patients compared with the BPPV cured $(P<0.05)$. Our results indicated that after maneuver intervention, patients' biological and physiological functions involving the repression of miR-34a expression and the activation of SIRT1, PGC-1 and PPAR- $\gamma$ were enhanced.

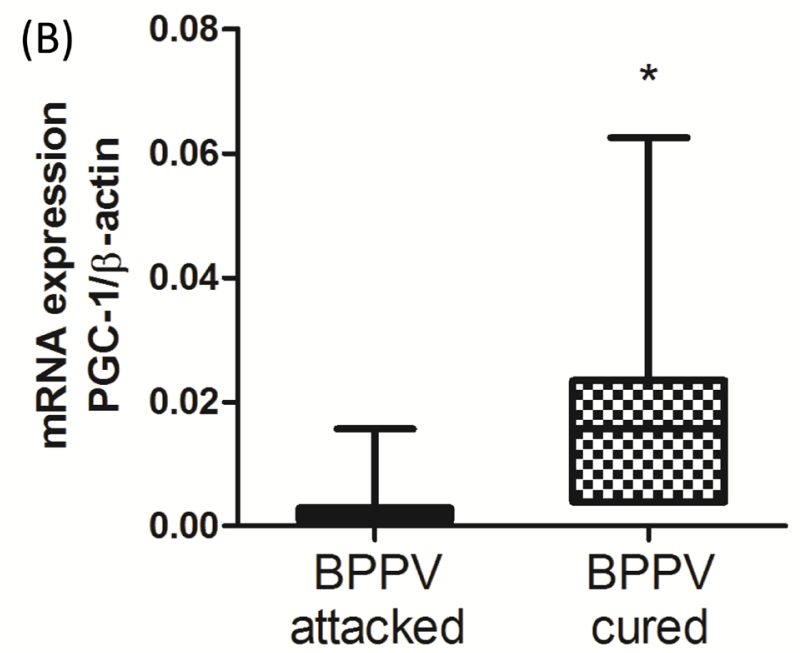

Figure 3: Expression levels of SIRT1 downstream genes in BPPV-attacked and BPPV-cured patients after treatment of maneuver exercise. Total blood mRNAs were isolated from patients, and the A. p54, B. PPAR- $\gamma$ and C. PGC-1 expression were tested before and after maneuver exercise intervention using real-time PCR. 
The expression of FoxO family genes was mitigated during BPPV episodes

It has been reported that the FoxO family of genes modulate a direct effect on hundreds of coupled sideand downstream genes [28]. FoxO genes are strongly associated with human longevity, aging and degeneration [29]. FoxO genes are important SIRT1 downstream targeting in regulation of cellular function by deacetylation of FoxO genes [30, 31]. We were interested to observe the expression levels of FoxOs in patients during BPPV episodes. As shown in Figure 4A and 4B, we found that FoxO1 and FoxO3 expression was significantly mitigated in BPPV attacked samples compared with BPPV cured samples $(P<0.05)$.

\section{The expression of FoxO family genes was mitigated during BPPV episodes}

During the aging process, genes involved in inflammatory responses are elevated in human brains [32]. Glass et al. suggested that the increase in SIRT1 expression inhibits pro-inflammatory responses, thus highlighting the potent anti-aging effects of neuronal degenerative diseases [33]. Nuclear factor kappa B (NF- $\mathrm{BB}$ ) is a key regulator of inflammatory pathways [34]. The NF- $\mathrm{kB}$ binding domain has been identified as the motif most highly involved with the aging process by bioinformatics

(A)

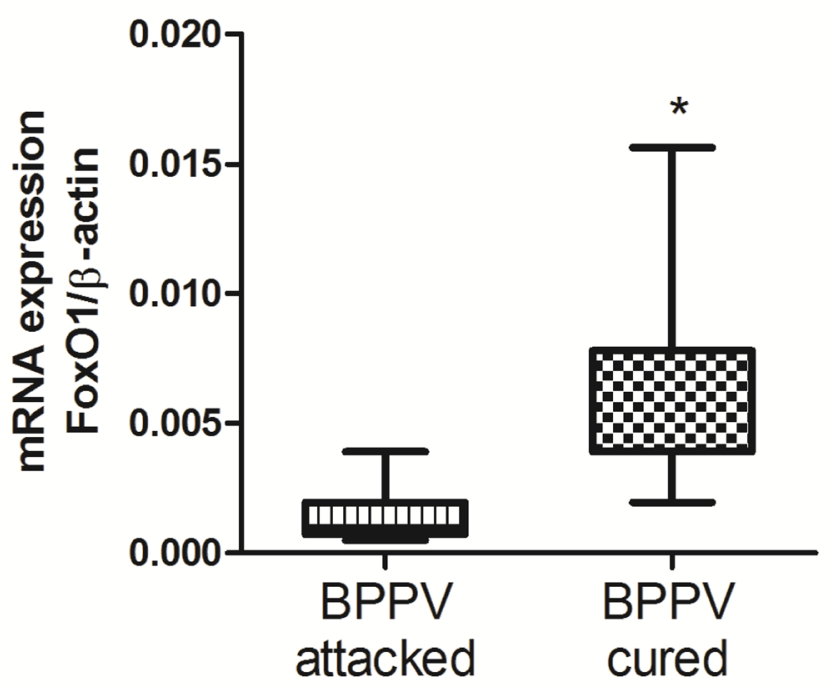

analyses [1]. Furthermore, COX-2 expression is known to be enriched during senescence, leading to an up-regulation of prostaglandin E2 (PGE2) production [35]. Activated $\mathrm{NF}-\kappa \mathrm{B}$ binds to COX-2 promoter region is necessary to facilitate COX-2 activation [36]. On investigating the levels of COX-2 and PGE2 in BPPV patient blood samples, we found that COX-2 mRNA expression was higher in the BPPV attacked samples than the BPPV cured samples (Figure 5A). We also confirmed that PGE2 concentrations were higher in the BPPV attacked samples than in the BPPV cured samples (Figure 5B). SIRT1 is considered as an important NF- $\mathrm{KB}$ inhibition, hence, the inhibitory ability of SIRT1 in anti-inflammation and reduction of pro-inflammatory responses [37] [38]. In Figure 6, we showed that IL-1, IL-6, IL-8 and NF- $\mathrm{BB}$ concentrations were significantly higher in the BPPV attacked samples by ELISA assays.

\section{Oxidative stress is increased in BPPV patients during BPPV episodes}

Reactive oxygen species (ROS) have been shown to be a major factor involved in the progression of neurodegenerative diseases [39]. An increase in lipid peroxidation and a decrease in polyunsaturated fatty acids have been confirmed in neurodegenerative diseases [40]. Mir-34a and SIRT1 have been shown to functionally regulate redox status. For example, mir-34a modulates

Figure 4: Expression levels of FoxOs genes in BPPV-attacked and BPPV-cured patients after treatment of maneuver exercise. Total blood mRNAs were isolated, and the A. FoxO1 and B. FoxO3 expression were observed before and after maneuver exercise intervention using real-time PCR. 
cell apoptosis by regulating ROS production and NOX2 expression [41], and SIRT1 inhibits ROS formation via mediating mitochondrial biogenesis [42]. We confirmed that hydrogen peroxide concentrations were relatively higher in BPPV- attacked samples than in BPPV-cured samples (Figure 7A). Benzi et al. reported that age-related changes in antioxidant function are involved in enzymatic and nonenzymatic events [43]. In Figure 7B, we shown the SOD activities were significantly reduced in patients with BPPV after treatment.

\section{DISCUSSION}

To date, the recommended treatment for BPPV remains to be the repositioning maneuvers/procedures [44].The dislodged calcium carbonate crystals, which presumably arise from within the semicircular canals, can be relocated back into the vestibule through this non-invasive CRP maneuver [45]. In our study, we demonstrated miR-34a levels were significantly higher and SIRT1 expression levels were significantly lower in blood samples of BPPV patients immediately after BPPV treatment (BPPV attacked) compared with the samples collected after completion of treatments. We also suggested that DBC1 is affected after the repositioning treatments of BPPV. Moreover, FoxO, PGC- $1 \alpha$, PPAR- $\gamma$ and p53 expression levels were also altered after recovery of BPPV. We proposed the hypothesis that following

(A)

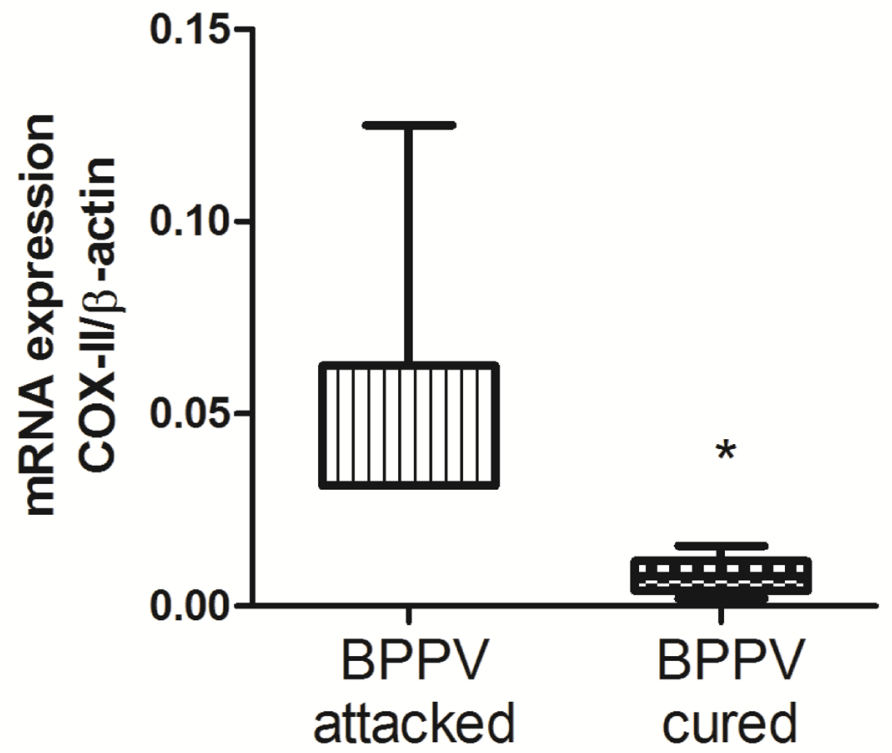

successful maneuver exercises, pro-inflammatory responses in the blood were corrected, and the oxidative stress in BPPV subjects were thus attenuated (Figure 8).

BPPV occurs with advancing age, and more frequently in females. Therefore, the etiology of BPPV may be partly due to degeneration [46]. The peak incidence of BPPV occurs in individuals between 50 and 70 years of age [4]. In patients $>70$ years of age, the occurrence and recurrence rates are significantly higher [5]. miRNAs in human blood samples have been considered a key class of biomarkers, as they have been shown to influence physiological conditions ranging from cancer to brain dysfunction. The level of circulating miR$34 \mathrm{a}$ in human plasma has been linked to Alzheimer's disease [13]. Increased miR-34a levels were also found in aged mouse brains [10]. In this study, we demonstrated for the first time that miR-34a expression levels were higher in the BPPV attacked samples; however, whether BPPV episodes are directly related to degeneration requires further investigation. The contribution of the miR-34a/ SIRT1 axis to senescence has been validated that miR34a functions to induce endothelial senescence via SIRT1 regulation [48]. SIRT1 has its protective roles in different neurodegenerative diseases, such as Alzheimer's disease and Parkinson's disease [47]. It has been well documented that one of the SIRT1 downstream gene, DBC1, is a main negative regulator of SIRT1 [24]. In addition, DBC1 is associated with IKK- $\beta$ to increase NF- $\kappa$ B transcriptional

Figure 5: Pro-inflammatory evens in BPPV-attacked and BPPV-cured patients after treatment of maneuver exercise. Total blood mRNAs were isolated, and the A. COX-II expression were observed before and after maneuver exercise intervention using realtime PCR Blood plasma was isolated from patients. B. PGE2 concentration werewere investigated before and after exercise intervention using ELISA kits. 
activities [25]. Calliari et al reported SIRT1 activation mitigates neurodegeneration through DBC1inhibition [48]. These finding suggest that DBC1 might play a key role in the mediation of neurodegenerative diseases. BPPV has been considered as a degenerative disease [49] and our study echoes that DBC1 mRNA and protein expression levels were attenuated following a BPPV cure.

A previous study indicated that collapse of mitochondrial activities may lead to the formation of neurodegenerative diseases [50]. One previous literature found that SIRT1 could activate PGC-1 via deacetylation in the mitochondria, thereby controlling mitochondrial biogenesis and oxidative phosphorylation [51]. PGC-1 activation is important for the facilitation of reactive oxygen species (ROS)-detoxifying enzymes. PGC-1 dysfunction activates oxidative stress and neurodegenerative disease [52]. In this study, we confirmed that blood PGC-1 and ROS levels can be

(A)

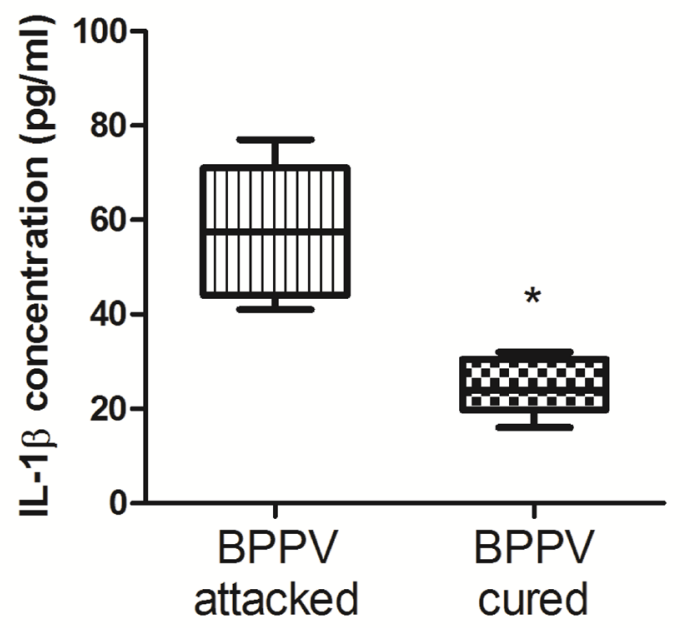

(C)

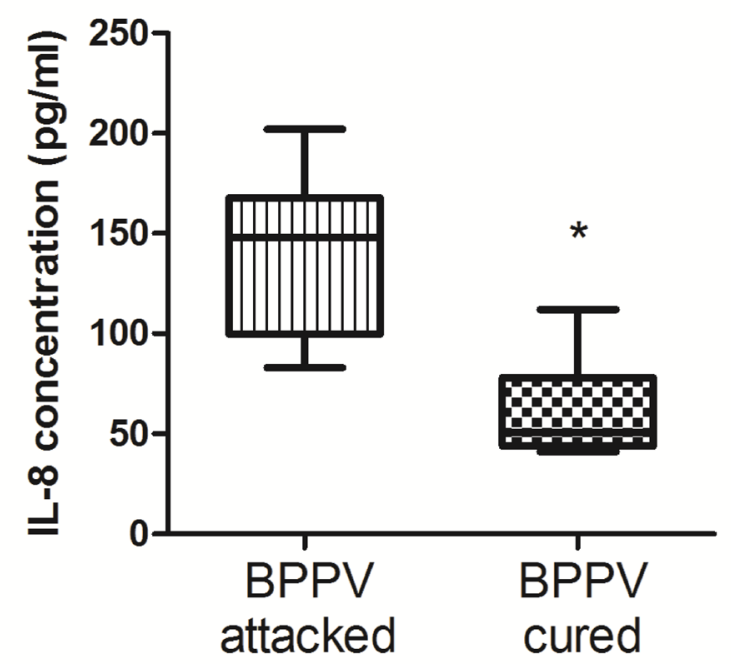

reduced after maneuver treatment in BPPV patients (Figure 3B and Figure 7A), indicating that mitochondrial mediated oxidation changes may be involved in the recovery of BPPV. The FoxO family has been correlated with aging and neuronal degenerative damage through modulation of SIRT1.FOXO up-regulates SOD and catalase, which delays axonal degeneration and neuronal loss [53]. Pino et al. also suggested that FoxO3 determines the accumulation of $\alpha$-synuclein and mediates the fate of dopaminergic neurons in the substantia nigra [54]. Taken together, the activation of FoxO family genes may be a novel tactic in the intervention of diseases related to degeneration, possibly in diseases like BPPV (In Figure 4).

BPPV is the most common form of vestibular dysfunction in the elderly [55]. This mechanism might involve the up-regulation of redox-sensitive transcription factors via the increasing effects of inflammatory injuries

(B)

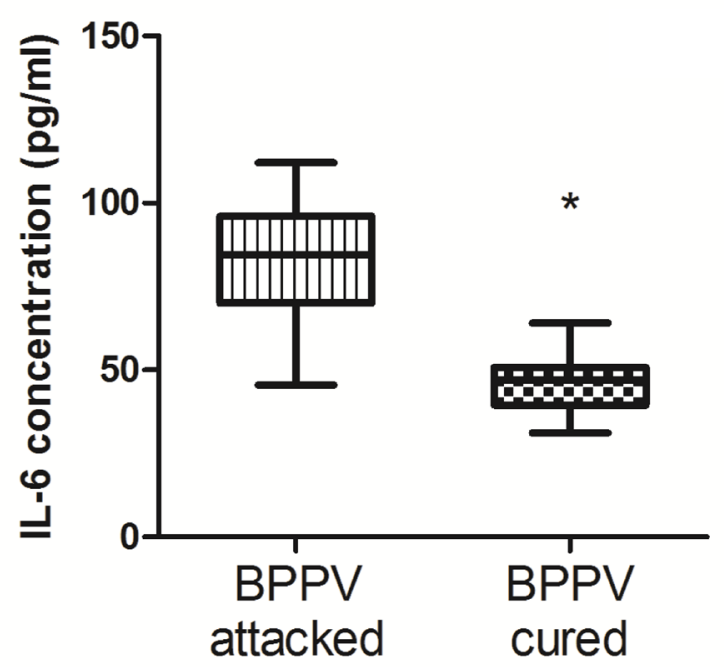

(D)

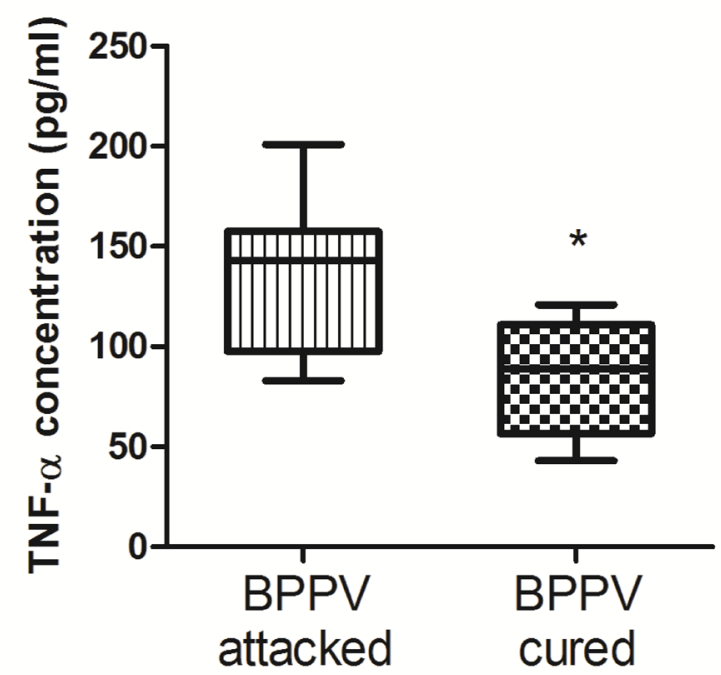

Figure 6: Pro-inflammatory cytokines concentration in BPPV-attacked and BPPV-cured patients after treatment of maneuver exercise. Blood plasma was isolated from patients. A. IL-1 $\beta$, B. IL-6, C. IL-8 and D. TNF- $\alpha$ were investigated before and after exercise intervention using ELISA kits. 

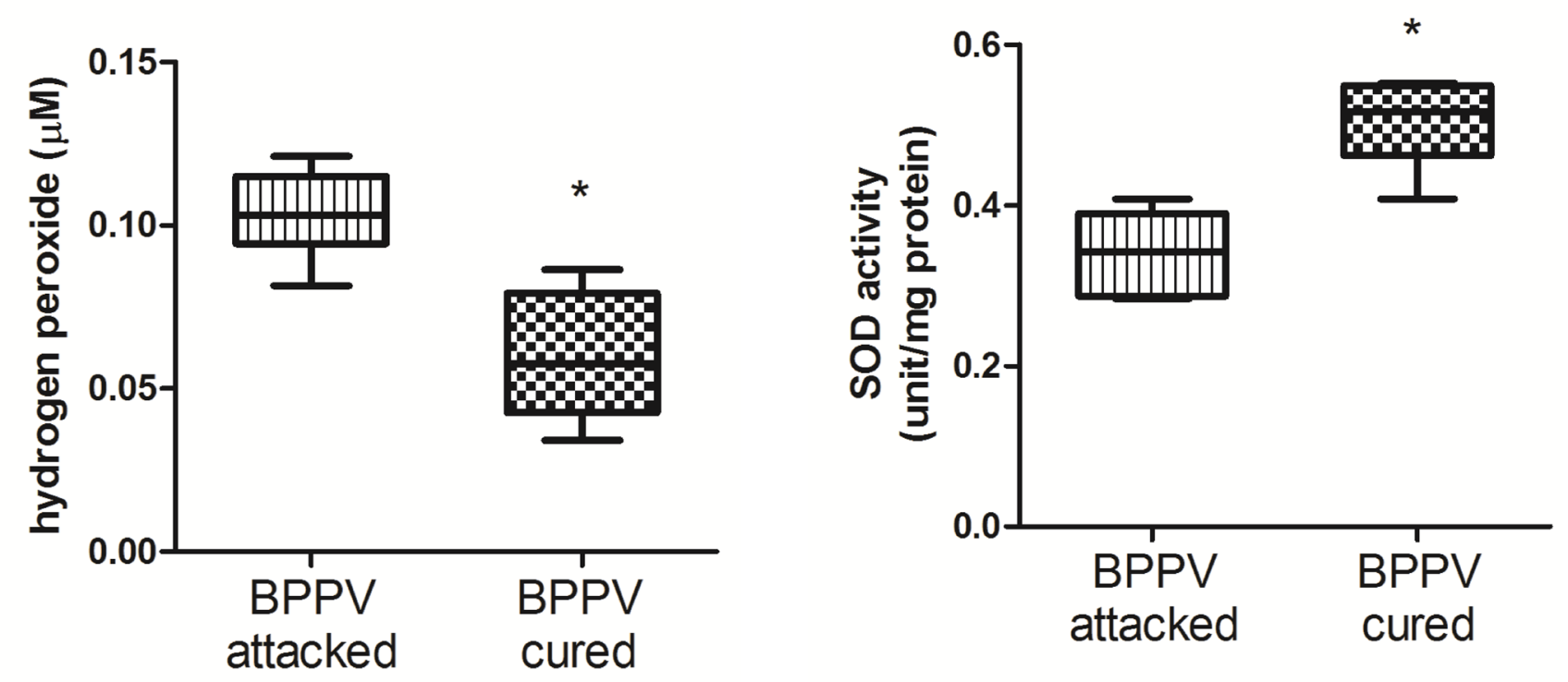

Figure 7: Oxidative status in BPPV-attacked and BPPV-cured patients after treatment of maneuver exercise. Plasma was isolated from whole blood and $\mathbf{A}$. $\mathrm{H} 2 \mathrm{O} 2$ concentration and $\mathbf{B}$. SOD activity were investigated using kits.

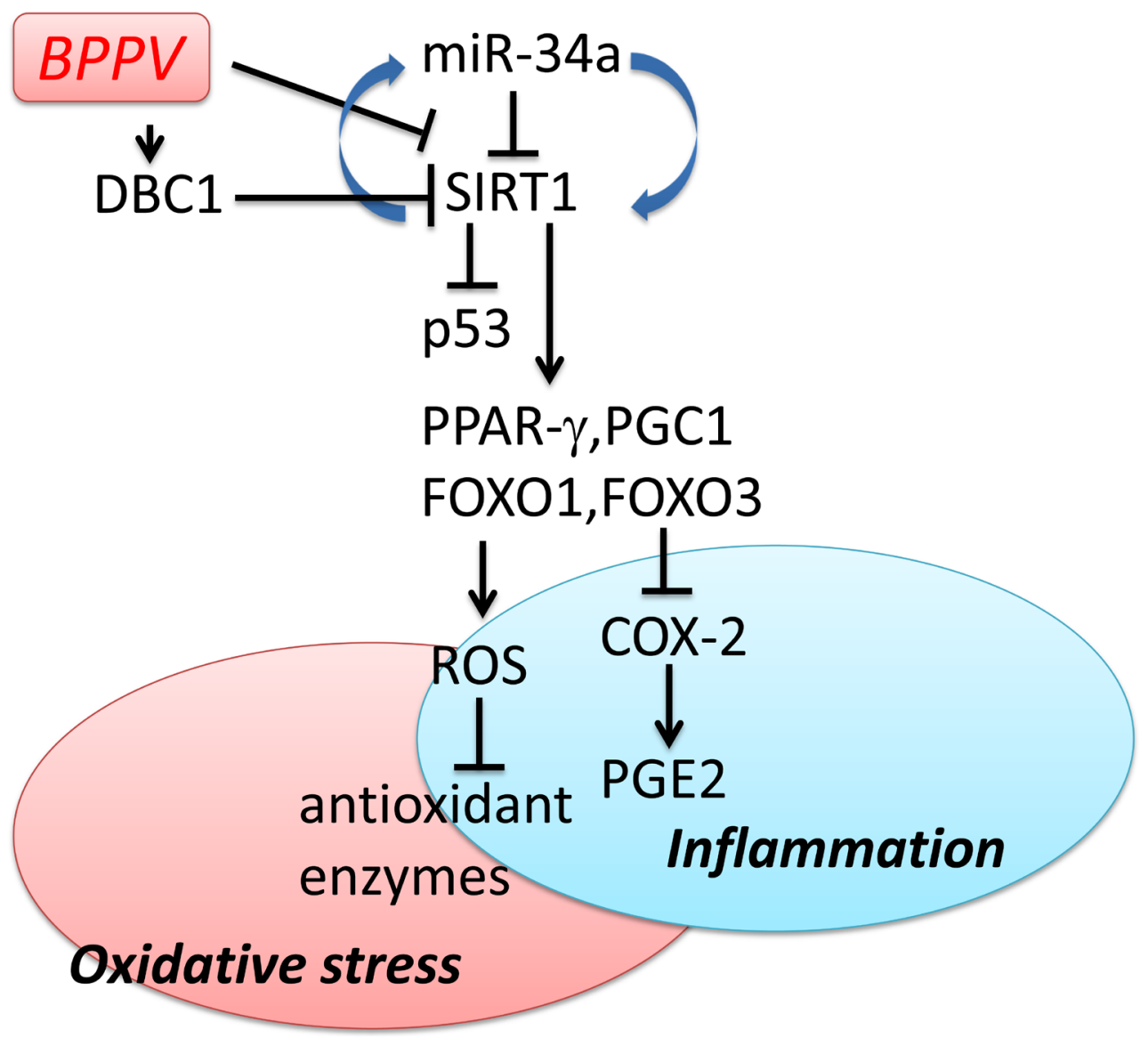

Figure 8: Schematic diagram showing the possible epigenetic regulation of BPPV by maneuver exercise. As depicted, maneuver exercise intervention reduces miR-34a and enhances SIRT1 expression and also up-regulates PPAR- $\gamma$, PGC-1 expression. Maneuver exercise intervention improves antioxidant enzyme activity and reduces the ROS concentration in blood. The $\rightarrow$ indicates activation or induction, and $\nmid$ indicates inhibition or blockade. 
during senescence [56]. Goto et al suggested that BPPV is correlated to oxidative stress and pro-inflammatory responses [57].SIRT1 can directly interact with p65 [58] and mitigate the transcription activity of activator protein-1 (AP-1), which inhibits the important proinflammatory gene cyclooxygenase-2 (COX-2) [59]. In Figure 5 and Figure 6, we revealed that COX-2 expression levels as well as pro-inflammatory cytokines PEG2, IL1, IL-6, IL-8 and TNF- $\alpha$ were significantly higher during BPPV attacks. These finding suggest that inflammation may be persistently activated in BPPV episodes. Previous bioinformatics findings revealed that miR-34a has many potential target genes, and cellular investigations have identified several of these targets, such as SIRT1 [11, 60]. Yamakuchi et al. reported that miR-34a targets the 3'UTR of SIRT1. The same report indicated that miR-34a inhibition reduces SIRT1 expression, thereby regulating cell apoptosis [11]. Interestingly, SIRT1 overexpression protected against pre-miR-34a-induced senescence in endothelial cells, indicating that miR-34a contributes to cellular senescence through the repression of SIRT1 function [12].

One limitation of our study is its cross-sectional design. A longitudinal study will be valuable to determine the roles of miR-34a/SIRT1 in long-term effects of CRP. Second, only blood and plasma samples were tested in this study, and therefore, our findings may not be able to reflect the exact physiological processes in human. However, our data has shed a light on the biomolecular changes in rehabilitation treatment of BPPV. Further parameters and tools are required in our future studies to further elucidate the pathophysiological conditions in CRP treatment. Moreover, we previously reported that the plasma of post-treatment BPPV patients had lower mean MDA levels than pretreatment samples. We also found that the mean levels of the antioxidative enzyme SOD were higher in plasma from post-treatment BPPV patients than their pretreatment samples. Notably, oxidative stress remained higher in BPPV post-treatment samples than samples from non-dizzy subjects despite the fact that the reduction in oxidative stress paralleled symptomatic relief in BPPV patients after CRP [61]. The expression levels of miR-34a and SIRT1 could also be compared between patients and healthy individuals to further validate the hypothesis that BPPV treatment might involve some epigenetic regulations through the mediation of miR-34a, SIRT1 functions and repression of redox status.

\section{MATERIALS AND METHODS}

\section{BPPV diagnosis and treatment}

Patients with chief complaints of positional vertigo were examined using the Dix-Hallpike test (sensitivity
$82 \%$, specificity $71 \%$ ) $[62,63]$. The site of semicircular canal involvement was determined by the direction of nystagmus observed in the infrared video fixation-block goggles (Micromedical Computerized Real Eyes infrared video Frenzel with single, pivotal camera, Micromedical Technologies, Illinois, USA). Patients with up and torsional beat nystagmus that lasted for less than 1 minute on the left or right side of the Dix-Hallpike position were included in this study. Therefore, only patients with posterior canal canalolithiasis BPPV [1] were included in this study. Patients were then treated with posterior CRP as described by Epley [64]. No mastoid vibration or any premedication was administered in our treatment procedures. Blood samples of $5 \mathrm{cc}$ were drawn immediately after the CRP (BPPV-attacked samples). Patients were also instructed to keep their head upright for 2 hours and avoid lying down on the side of involvement. A follow up visit was arranged 1-2 weeks after the CRP (BPPV cured samples). A complete cure was defined as being symptom free and the absence of nystagmus in the Hallpike-Dix position. Blood samples (5 cc) were then drawn after the confirmation of a complete BPPV cure. All patients signed an informed consent form approved by Taipei Veterans General Hospital Institutional Review Board prior to the first blood sampling.

\section{Isolation of mRNA and quantitative real-time PCR}

BPPV patient blood was collected in BD K2EDTA tubes $(5 \mathrm{ml})$ after maneuver intervention. The RNeasy Plus mini kit (Qiagen, Hilden, Germany) was used to isolate total blood RNA. The Experion Automated Electrophoresis Station (Bio-Rad) was used to check RNA quality. In this study, we used a homology search within the human genome (BLAST, National Center for Biotechnology Information, Bethesda, MD, USA) to determine oligonucleotide specificity, and the primers were confirmed by dissociation curve analysis. The oligonucleotide sequences are listed in Table 1. Oligonucleotides for SIRT1, DBC1, PGC-1a, p53, FoxO1, Foxo3, COX-2 and $\beta$-actin were designed using the computer software package Primer Express 2.0 (Applied Biosystems, Foster City, CA, USA). All oligonucleotides were synthesized by Invitrogen (Breda, The Netherlands). SYBR Green and an ABI 7000 sequence detection system (Applied Biosystems) were used to perform PCR according to the manufacturer's guidelines.

\section{Investigation of microRNA expression levels}

Blood miRNAs were isolated by PAXgene Blood miRNA Kit. Primers specific for miR-34a were obtained from Applied Biosystems (Foster City, CA, USA) to execute quantitative real-time PCR according to the 
Table 1: The oligonucleotide sequences for real-time PCR assay.

\begin{tabular}{|c|c|c|}
\hline Gene & Sense & Anti-sense \\
\hline SIRT1 & 5'-TGTGGTAGAGCTTGCATTGATCTT-3' & 5'-GGCCTGTTGCTCTCCTCAT-3' \\
\hline DBC1 & 5'-CCC TCG CCC GCC TAC TAT-3' & 5'-GCT GGG CGG GGT TGT AGA-3' \\
\hline p53 & 5'-GCCCACTTCACCGTACTAA -3' & 5'-TGGTTTCAAGGCCAGATGT-3' \\
\hline PGC-1 & 5'-CCGCACGCACCG AAA-3' & 5'-TCGTGCTGATATTCCTCGTAGCT-3' \\
\hline PPAR- $\gamma$ & $\begin{array}{c}\text { 5'-AGTGTGAATTACAGC } \\
\text { AAATCTCTGTTTT-3' }\end{array}$ & 5'-GCACCATGCTCTGGGTCA A-3' \\
\hline Fox01 & 5'-ATGGTCAAGAGCGTGCCC-3' & 5'-GATTGAGCATCCACCAAG-3' \\
\hline FoxO3 & 5'-TCTCCCGTCAGCCAGTCTAT-3' & 5'-AGTCACTGGGGA ACTTGTCG-3' \\
\hline$\beta$-actin & 5'-CGGGAAATCGTGCGTGAC-3' & 5'-TGCCCAGGAAGGAAGGCT-3' \\
\hline miR-34a & 5'-CGGTATCATTTGGCAGTGTCT-3' & 5'-GTGCAGGGTCCGAGGT-3' \\
\hline U6 & 5'-GCCCGCTAGCTTATCAGACTGATG-3' & 5'-GTGCAGGGTCCGAGGT-3' \\
\hline
\end{tabular}

vendor's protocol for TaqMan miRNA assays. Isolated total RNA from human plasma was initially processed for reverse transcription (RT) using two miRNA-specific primers (miR-34a: AB Assay ID 000426) to obtain RT products that were subsequently used for quantitative real-time PCR on a 7500 Fast System Real-Time PCR cycler (Applied Biosystems). U6 was used as a control to calculate the relative expression levels of miR-34a. Numerical indices of these expression levels are expressed using the $1 / \Delta \mathrm{CT}$ method, and the values for each individual microRNA were obtained after subtracting the CT value of snoRNA202 (AB assay ID 001232, Applied Biosystems.

\section{ELISA assay for cytokines}

After collection of human plasma from whole blood after $2500 \mathrm{x}$ g centrifuge at $4^{\circ} \mathrm{C}$ for $10 \mathrm{~min}$. Human plasma was collected from whole blood after centrifugation at $2500 \mathrm{~g}$ at $4^{\circ} \mathrm{C}$ for $10 \mathrm{~min}$. PEG2, IL-1beta, IL-6, IL-8 and TNF- $\alpha$ plasma concentrations were measured using an ELISA kit obtained from R\&D Systems (Minneapolis, MN, USA). DBC1 plasma concentration was tested by as ELISA kit obtained from MyBioSource (San Diego, CA, USA)

\section{SOD activity measurement}

After collection of human plasma from whole blood after 2500x g centrifuge at $4^{\circ} \mathrm{C}$ for $10 \mathrm{~min}$. The SOD activity assay kit (Cell Biolabs, STA-340) was determined $v i a$ an enzymatic assay method using a commercial kit according to the manufacturer's instructions. Enzymatic activity was revealed to units per milligram of protein.

\section{Hydrogen peroxide concentration assay}

After collection of human plasma from whole blood after $2500 \mathrm{x}$ g centrifuge at $4^{\circ} \mathrm{C}$ for $10 \mathrm{~min}$. The hydrogen peroxide assay kit $\left(\mathrm{H}_{2} \mathrm{O}_{2}\right.$; Cell Biolabs, STA-344) was used to test the $\mathrm{H}_{2} \mathrm{O}_{2}$ concentration. The plasma concentration of hydrogen peroxide was shown to $\mu \mathrm{M}$ according to the manufacturer's instructions of the protocol.

\section{Statistical analyses}

The data are expressed as the mean $\pm \mathrm{SD}$. The experimental results were analyzed using Paired sample $t$-test to compare hematological mRNA levels and plasma parameters. The significance level was set at $P<0.05$.

\section{ACKNOWLEDGMENTS}

This study was funded by NSC-96-2314-B-075056-MY3, 100-2627-B-075-002-, 101-2627-B-075-001-, MOST 102-2314-B-010-061-MY3, from the Ministry of Science and Technology; MOST (former National Science Council; NSC) ; V104C-030,V105C-151 and DV102- 
10 from Taipei Veterans General Hospital-National Yang-Ming University Excellent Physician Scientists Cultivation Program, Taiwan.

\section{CONFLICTS OF INTERESTS}

The authors declare no actual or potential conflicts of interests.

\section{REFERENCES}

1. Hall SF, Ruby RR and McClure JA. The mechanics of benign paroxysmal vertigo. J Otolaryngol. 1979; 8:151-158.

2. Heidenreich KD, Carender WJ, Heidenreich MJ and Telian SA. Strategies to distinguish benign paroxysmal positional vertigo from rotational vertebrobasilar ischemia. Annals of vascular surgery. 2010; 24:553 e551-555.

3. Otsuka K, Suzuki M, Shimizu S, Konomi U, Inagaki T, Iimura Y, Hayashi M and Ogawa Y. Model experiments of otoconia stability after canalith repositioning procedure of BPPV. Acta oto-laryngologica. 2010; 130:804-809.

4. von Brevern M, Radtke A, Lezius F, Feldmann M, Ziese $\mathrm{T}$, Lempert $\mathrm{T}$ and Neuhauser H. Epidemiology of benign paroxysmal positional vertigo: a population based study. J Neurol Neurosurg Psychiatry. 2007; 78:710-715.

5. Kao CL, Hsieh WL, Chern CM, Chen LK, Lin MH and Chan RC. Clinical features of benign paroxysmal positional vertigo (BPPV) in Taiwan: differences between young and senior age groups. Arch Gerontol Geriatr. 2009; 49 Suppl 2:S50-54.

6. Brandt $T$ and Daroff RB. Physical therapy for benign paroxysmal positional vertigo. Arch Otolaryngol. 1980; 106:484-485.

7. Semont A, Freyss G and Vitte E. Curing the BPPV with a liberatory maneuver. Advances in oto-rhino-laryngology. 1988; 42:290-293.

8. Epley JM. Human experience with canalith repositioning maneuvers. Annals of the New York Academy of Sciences. 2001; 942:179-191.

9. Suarez-Gomez M, Alejandre-Duran E and Ruiz-Rubio M. MicroRNAs in bipolar disorder: diagnostic and therapeutic applications [Article in Spanish]. Revista de neurologia. 2011; 53:91-98.

10. Li X, Khanna A, Li N and Wang E. Circulatory miR34a as an RNAbased, noninvasive biomarker for brain aging. Aging (Albany NY). 2011; 3:985-1002. doi:10.18632/ aging.100371

11. Yamakuchi M, Ferlito M and Lowenstein CJ. miR-34a repression of SIRT1 regulates apoptosis. Proceedings of the National Academy of Sciences of the United States of America. 2008; 105:13421-13426.

12. Ito T, Yagi S and Yamakuchi M. MicroRNA-34a regulation of endothelial senescence. Biochemical and biophysical research communications. 2010; 398:735-740.
13. Bhatnagar S, Chertkow H, Schipper HM, Yuan Z, Shetty V, Jenkins S, Jones $\mathrm{T}$ and Wang E. Increased microRNA-34c abundance in Alzheimer's disease circulating blood plasma. Frontiers in molecular neuroscience. 2014; 7:2.

14. Fyrmpas G, Barkoulas E, Haidich AB and Tsalighopoulos M. Vertigo during the Epley maneuver and success rate in patients with BPPV. European archives of oto-rhinolaryngology. 2013; 270:2621-2625.

15. Kim HJ, Jung KJ, Yu BP, Cho CG, Choi JS and Chung HY. Modulation of redox-sensitive transcription factors by calorie restriction during aging. Mechanisms of ageing and development. 2002; 123:1589-1595.

16. Chang J, Rimando A, Pallas M, Camins A, Porquet D, Reeves J, Shukitt-Hale B, Smith MA, Joseph JA and Casadesus G. Low-dose pterostilbene, but not resveratrol, is a potent neuromodulator in aging and Alzheimer's disease. Neurobiology of aging. 2012; 33:2062-2071.

17. Baek BS, Kwon HJ, Lee KH, Yoo MA, Kim KW, Ikeno Y, Yu BP and Chung HY. Regional difference of ROS generation, lipid peroxidation, and antioxidant enzyme activity in rat brain and their dietary modulation. Archives of pharmacal research. 1999; 22:361-366.

18. Beharka AA, Wu D, Han SN and Meydani SN. Macrophage prostaglandin production contributes to the age-associated decrease in $\mathrm{T}$ cell function which is reversed by the dietary antioxidant vitamin E. Mechanisms of ageing and development. 1997; 93:59-77.

19. Salminen A, Ojala J, Huuskonen J, Kauppinen A, Suuronen $\mathrm{T}$ and Kaarniranta $\mathrm{K}$. Interaction of aging-associated signaling cascades: inhibition of NF-kappaB signaling by longevity factors FoxOs and SIRT1. Cellular and molecular life sciences. 2008; 65:1049-1058.

20. Kim JS, Oh SY, Lee SH, Kang JH, Kim DU, Jeong SH, Choi KD, Moon IS, Kim BK, Oh HJ and Kim HJ. Randomized clinical trial for apogeotropic horizontal canal benign paroxysmal positional vertigo. Neurology. 2012; 78:159-166.

21. Pallas M, Pizarro JG, Gutierrez-Cuesta J, Crespo-Biel N, Alvira D, Tajes M, Yeste-Velasco M, Folch J, Canudas AM, Sureda FX, Ferrer I and Camins A. Modulation of SIRT1 expression in different neurodegenerative models and human pathologies. Neuroscience. 2008; 154:13881397.

22. Herskovits AZ and Guarente L. SIRT1 in Neurodevelopment and Brain Senescence. Neuron. 2014; 81:471-483.

23. Kim W and Kim JE. Deleted in breast cancer 1 (DBC1) deficiency results in apoptosis of breast cancer cells through impaired responses to UV-induced DNA damage. Cancer letters. 2013; 333:180-186.

24. Kim JE, Chen J and Lou Z. DBC1 is a negative regulator of SIRT1. Nature. 2008; 451:583-586.

25. Park SH, Riley Pt and Frisch SM. Regulation of anoikis by deleted in breast cancer-1 (DBC1) through NF-kappaB. 
Apoptosis. 2013; 18:949-962.

26. Cheng J, Cui R, Chen $\mathrm{CH}$ and $\mathrm{Du}$ J. Oxidized lowdensity lipoprotein stimulates p53-dependent activation of proapoptotic Bax leading to apoptosis of differentiated endothelial progenitor cells. Endocrinology. 2007; 148:2085-2094.

27. Min SW, Sohn PD, Cho SH, Swanson RA and Gan L. Sirtuins in neurodegenerative diseases: an update on potential mechanisms. Frontiers in aging neuroscience. 2013; 5:53.

28. Willcox BJ, Donlon TA, He Q, Chen R, Grove JS, Yano $\mathrm{K}$, Masaki KH, Willcox DC, Rodriguez B and Curb JD. FOXO3A genotype is strongly associated with human longevity. Proceedings of the National Academy of Sciences of the United States of America. 2008; 105:1398713992.

29. Zheng X, Yang Z, Yue Z, Alvarez JD and Sehgal A. FOXO and insulin signaling regulate sensitivity of the circadian clock to oxidative stress. Proceedings of the National Academy of Sciences of the United States of America. 2007; 104:15899-15904.

30. Brunet A, Sweeney LB, Sturgill JF, Chua KF, Greer PL, Lin Y, Tran H, Ross SE, Mostoslavsky R, Cohen HY, Hu LS, Cheng HL, Jedrychowski MP, Gygi SP, Sinclair DA, Alt FW, et al. Stress-dependent regulation of FOXO transcription factors by the SIRT1 deacetylase. Science. 2004; 303:2011-2015.

31. Kobayashi Y, Furukawa-Hibi Y, Chen C, Horio Y, Isobe $\mathrm{K}$, Ikeda $\mathrm{K}$ and Motoyama N. SIRT1 is critical regulator of FOXO-mediated transcription in response to oxidative stress. Int J Mol Med. 2005; 16:237-243.

32. Lu T, Pan Y, Kao SY, Li C, Kohane I, Chan J and Yankner BA. Gene regulation and DNA damage in the ageing human brain. Nature. 2004; 429:883-891.

33. Glass CK, Saijo K, Winner B, Marchetto MC and Gage FH. Mechanisms underlying inflammation in neurodegeneration. Cell. 2010; 140:918-934.

34. Baker RG, Hayden MS and Ghosh S. NF-kappaB, inflammation, and metabolic disease. Cell metabolism. $2011 ; 13: 11-22$

35. Zdanov S, Bernard D, Debacq-Chainiaux F, Martien S, Gosselin K, Vercamer C, Chelli F, Toussaint O and Abbadie C. Normal or stress-induced fibroblast senescence involves COX-2 activity. Experimental cell research. 2007; 313:3046-3056.

36. Yamamoto K, Arakawa T, Ueda N and Yamamoto S. Transcriptional roles of nuclear factor kappa B and nuclear factor-interleukin-6 in the tumor necrosis factor alphadependent induction of cyclooxygenase-2 in MC3T3-E1 cells. The Journal of biological chemistry. 1995; 270:3131531320 .

37. Laveti D, Kumar M, Hemalatha R, Sistla R, Naidu VG, Talla V, Verma V, Kaur N and Nagpal R. Antiinflammatory treatments for chronic diseases: a review.
Inflammation \& allergy drug targets. 2013; 12:349-361.

38. Chen XP, Li MH, Cong ML, Kang YJ, Guo WP and Zhang YZ. SIRT1 inhibits IL-1beta mRNA transcription in lipopolysaccharide tolerant THP-1 cells [Article in Chinese]. Zhonghua liu xing bing xue za zhi. 2011; 32:613616.

39. Floyd RA. Neuroinflammatory processes are important in neurodegenerative diseases: an hypothesis to explain the increased formation of reactive oxygen and nitrogen species as major factors involved in neurodegenerative disease development. Free radical biology \& medicine. 1999; 26:1346-1355.

40. Markesbery WR. Oxidative stress hypothesis in Alzheimer's disease. Free radical biology \& medicine. 1997; 23:134147.

41. Li SZ, Hu YY, Zhao J, Zhao YB, Sun JD, Yang YF, Ji CC, Liu ZB, Cao WD, Qu Y, Liu WP, Cheng G and Fei Z. MicroRNA-34a induces apoptosis in the human glioma cell line, A172, through enhanced ROS production and NOX2 expression. Biochemical and biophysical research communications. 2014; 444:6-12.

42. Ye Q, Ye L, Xu X, Huang B, Zhang X, Zhu Y and Chen $X$. Epigallocatechin-3-gallate suppresses 1-methyl-4phenyl-pyridine-induced oxidative stress in $\mathrm{PC} 12$ cells via the SIRT1/PGC-1alpha signaling pathway. BMC complementary and alternative medicine. 2012; 12:82.

43. Benzie IF and Tomlinson B. Antioxidant power of angiotensin-converting enzyme inhibitors in vitro. British journal of clinical pharmacology. 1998; 45:168-169.

44. Froehling DA, Silverstein MD, Mohr DN, Beatty CW, Offord KP and Ballard DJ. Benign positional vertigo: incidence and prognosis in a population-based study in Olmsted County, Minnesota. Mayo Clinic proceedings. 1991; 66:596-601.

45. Epley JM. Positional vertigo related to semicircular canalithiasis. Otolaryngology--head and neck surgery. 1995; 112:154-161.

46. Hong SM, Park DC, Yeo SG and Cha CI. Vestibular evoked myogenic potentials in patients with benign paroxysmal positional vertigo involving each semicircular canal. American journal of otolaryngology. 2008; 29:184-187.

47. Duan W. Sirtuins: from metabolic regulation to brain aging. Frontiers in aging neuroscience. 2013; 5:36.

48. Calliari A, Bobba N, Escande C and Chini EN. Resveratrol delays Wallerian degeneration in a $\mathrm{NAD}(+)$ and $\mathrm{DBC} 1$ dependent manner. Experimental neurology. 2014; 251:91100.

49. Yang WS, Kim SH, Lee JD and Lee WS. Clinical significance of vestibular evoked myogenic potentials in benign paroxysmal positional vertigo. Otology \& neurotology. 2008; 29:1162-1166.

50. Lin MT and Beal MF. Mitochondrial dysfunction and oxidative stress in neurodegenerative diseases. Nature. 2006; 443:787-795. 
51. Rodgers JT, Lerin C, Haas W, Gygi SP, Spiegelman BM and Puigserver P. Nutrient control of glucose homeostasis through a complex of PGC-1alpha and SIRT1. Nature. 2005; 434:113-118.

52. St-Pierre J, Drori S, Uldry M, Silvaggi JM, Rhee J, Jager S, Handschin C, Zheng K, Lin J, Yang W, Simon DK, Bachoo $\mathrm{R}$ and Spiegelman BM. Suppression of reactive oxygen species and neurodegeneration by the PGC-1 transcriptional coactivators. Cell. 2006; 127:397-408.

53. Calixto A, Jara JS and Court FA. Diapause formation and downregulation of insulin-like signaling via DAF-16/ FOXO delays axonal degeneration and neuronal loss. PLoS genetics. 2012; 8:e1003141.

54. Pino E, Amamoto R, Zheng L, Cacquevel M, Sarria JC, Knott GW and Schneider BL. FOXO3 determines the accumulation of alpha-synuclein and controls the fate of dopaminergic neurons in the substantia nigra. Human molecular genetics. 2014; 23:1435-1452.

55. Iwasaki S and Yamasoba T. Dizziness and Imbalance in the Elderly: Age-related Decline in the Vestibular System. Aging and disease. 2015; 6:38-47.

56. Kabe Y, Ando K, Hirao S, Yoshida M and Handa H. Redox regulation of NF-kappaB activation: distinct redox regulation between the cytoplasm and the nucleus. Antioxidants \& redox signaling. 2005; 7:395-403.

57. Goto F, Hayashi K, Kunihiro T and Ogawa K. The possible contribution of angiitis to the onset of benign paroxysmal positional vertigo (BPPV). The international tinnitus journal. 2010; 16:25-28.

58. Chen L, Fischle W, Verdin E and Greene WC. Duration of nuclear NF-kappaB action regulated by reversible acetylation. Science. 2001; 293:1653-1657.
59. Gao Z and Ye J. Inhibition of transcriptional activity of c-JUN by SIRT1. Biochem Biophys Res Commun. 2008; 376:793-796.

60. Yamakuchi M and Lowenstein CJ. MiR-34, SIRT1 and p53: the feedback loop. Cell Cycle. 2009; 8:712-715.

61. Tsai KL, Cheng YY, Leu HB, Lee YY, Chen TJ, Liu DH and Kao CL. Investigating the role of Sirt1-modulated oxidative stress in relation to benign paroxysmal positional vertigo and Parkinson's disease. Neurobiology of aging. $2015 ; 36: 2607-2616$.

62. Dix MR and Hallpike CS. The pathology symptomatology and diagnosis of certain common disorders of the vestibular system. Proceedings of the Royal Society of Medicine. 1952; 45:341-354.

63. Lopez-Escamez JA, Lopez-Nevot A, Gamiz MJ, Moreno PM, Bracero F, Castillo JL and Salinero J. Diagnosis of common causes of vertigo using a structured clinical history [Article in Spanish]. Acta otorrinolaringologica espanola. 2000; 51:25-30.

64. Epley JM. The canalith repositioning procedure: for treatment of benign paroxysmal positional vertigo. Otolaryngology--head and neck surgery. 1992; 107:399404. 